\title{
BDNF protects retinal neurons from hyperglycemia through the TrkB/ERK/MAPK pathway
}

\author{
$\mathrm{YU} \mathrm{LIU}^{1}$, LIJIAN TAO ${ }^{2}, \mathrm{XIAO} \mathrm{FU}^{2}$, YINGCHUN ZHAO $^{3}$ and XUELIANG XU ${ }^{4}$ \\ ${ }^{1}$ Department of Ophthalmology, The Third Xiangya Hospital of Central South University, Changsha, Hunan 410013; \\ ${ }^{2}$ Department of Nephrology, Xiangya Hospital, Changsha, Hunan 410008, P.R. China; \\ ${ }^{3}$ Department of Biostatistics and Bioinformatics, Tulane University School of Public Health and Tropical Medicine, \\ New Orleans, LA 70112, USA; ${ }^{4}$ Department of Ophthalmology, Xiangya Hospital, Changsha, Hunan 410008, P.R. China
}

Received December 9, 2012; Accepted April 11, 2013

DOI: $10.3892 / \mathrm{mmr} .2013 .1433$

\begin{abstract}
Diabetic retinopathy (DR) is one of the most common diabetic eye diseases and a leading cause of blindness. It is characterized by changes in the blood vessels of the retina. The pathogenesis of DR is complex and to date, the precise mechanisms involved remain unclear. Previous studies have reported that DR is associated with neurodegeneration and that apoptosis may occur in diabetic retinas. In the present study, retinal neurons under conditions of hyperglycemia were used as a model to study apoptosis in diabetic retinas. Retinal neurons exposed to hyperglycemia exhibited high levels of apoptosis. Brain-derived neurotrophic factor (BDNF), a member of the neurotrophin family, was effective in protecting retinal neurons from hyperglycemia in vitro. BDNF promoted neuronal cell survival in a concentration-dependent manner. In addition, BDNF was demonstrated to promote the expression of tropomyosin-related kinase B (TrkB) and elevate the phosphorylation levels of TrkB and ERK in retinal neurons exposed to hyperglycemia. The results of the present study demonstrated that BDNF may protect retinal neurons from hyperglycemia via the TrkB/ERK/MAPK pathway and provides novel insights into the pathogenesis of DR.
\end{abstract}

\section{Introduction}

Diabetic retinopathy (DR), a leading cause of blindness worldwide, is an important area of study in clinical and basic research. The pathogenesis of DR is complex and to date, the precise mechanisms involved remain unclear. Previous studies have largely focused on the mechanisms underlying microvascular changes. However, an increasing number of studies

Correspondence to: Professor Xueliang Xu, Department of Ophthalmology, Xiangya Hospital, No. 87 Xiangya Road, Changsha, Hunan 410008, P.R. China

E-mail: xuxueliang8341@yahoo.com.cn

Key words: brain-derived neurotrophic factor, retinal neurons, apoptosis, diabetic retinopathy, ERK/MAPK signaling pathway have reported that the pathogenesis of DR correlates with neurodegeneration of the retina (1). Neuronal and glial tissues, which are sensitive to hyperglycemia, may be involved in the pathogenesis of DR. Microvascular damage is also likely to be associated with malfunctions of glial cell metabolism (2). Therefore, understanding the pathological changes which correlate with DR and the mechanisms that protect neurons, are likely to be important aims of future studies. Studies in animal models have demonstrated that at early stages of DR, specific retinal ganglion cells (RGCs) undergo apoptosis and retinal neurodegeneration is likely to be associated with a lack of brain-derived neurotrophic factor (BDNF) (3).

BDNF is a member of the neurotrophin family of growth factors and is important in the development, differentiation and maintenance of neurons. Previous studies have revealed that BDNF is critical for photoreceptor cells and the repair of damage to the retina and the optic nerve. BDNF promotes survival in injured RGCs induced by axotomy or retinal ischemia, and also promotes regeneration of the nerve fiber $(4,5)$. In addition, BDNF promotes the survival of retinal interneurons and is important for establishing phenotypes and synaptic connections in the developing retina (6). BDNF has been reported to inhibit neuroretinal cell death under conditions of ischemia and hypoxia, and to inhibit apoptosis in rat RGCs at early stages of DR (7). However, the mechanisms by which BDNF regulates RGCs remain unclear (8-10).

Tropomyosin-related kinase B (TrkB) is a receptor protein involved in the development and maturation of the central and peripheral nervous systems. BDNF has a high affinity for TrkB and p75 enhances the interaction between BDNF and TrkB (11). Upon ligand-binding, TrkB undergoes homodimerization, autophosphorylation and activation. It then recruits and activates several downstream effectors to regulate gene expression and protect neurons. Members of the TrkB downstream signaling cascade, including ERK/MAPK and PI3K/PKB, have been reported to be responsive to $\operatorname{BDNF}(12,13)$. Several studies have hypothesized that BDNF largely activates the ERK/MAPK pathway (14-17). By contrast, other studies have reported that activation of the ERK/MAPK pathway leads to cell death and PI3K/PKB is the main pathway involved in the protection of neurons induced by BDNF (18). In addition, it has been reported that both pathways are critical for neuropro- 
tection induced by BDNF. To the best of our knowledge, no studies to date have addressed which pathway dominates the response to TrkB activation $(13,19,20)$.

A number of studies have demonstrated in vivo that the death of retinal neurons at early stages of DR correlates with decreased levels of BDNF, and that TrkB is important for the protection of brain neurons and RGCs induced by BDNF. However, it is not known whether BDNF protects retinal neurons exposed to hyperglycemia in vitro or whether the ERK/MAPK pathway is activated in response to BDNF-induced neuroprotection.

The aim of the present study was to determine whether conditions of hyperglycemia increase the levels of apoptosis in retinal neurons in vitro and whether BDNF is effective in protecting retinal neurons from hyperglycemia. In addition, we determined whether BDNF promotes TrkB expression and activates the TrkB/ERK/MAPK pathway to decrease levels of apoptosis. This study is likely to provide novel insights into the pathogenesis of DR and be useful for the development of novel treatment strategies for this disease.

\section{Materials and methods}

Cell culture. Retinal neurons dissected from postnatal Wistar rats were grown in Neurobasal medium containing $5 \mathrm{mM}$ glucose, $4 \mathrm{mM}$ L-glutamine, $1.5 \mathrm{~g} / 1$ sodium bicarbonate, $1.0 \mathrm{mM}$ sodium pyruvate and B-27 serum-free supplements (all purchased from Gibco-BRL, Carlsbad, CA, USA). Retinal neurons were cultured in a $\mathrm{CO}_{2}$ incubator with $5 \% \mathrm{CO}_{2}$ at $37^{\circ} \mathrm{C}$. Cells were used at $80 \%$ confluence for the experiments. The study was approved by the ethics committee of the Ministry of Science and Technology of China, Beijing, China.

Immunohistochemistry. Immunohistochemistry assays were performed to investigate the effect of BNDF on TrkB protein expression. Briefly, retinal neuron cells were treated with BDNF (Sigma-Aldrich, St. Louis, MO, USA) at various concentrations 2 or 3 days following dissection, followed by the addition of $35 \mathrm{mmol} / \mathrm{l}$ glucose or buffer 1 day later. The cells were divided into 5 groups: i) $5.5 \mathrm{mmol} / 1$ glucose in basal medium (Ctr); ii) $35 \mathrm{mmol} / 1$ glucose (HG); iii) $35 \mathrm{mmol} / \mathrm{l}$ glucose + $75 \mathrm{ng} / \mathrm{ml} \mathrm{BDNF}(\mathrm{HG}+\mathrm{L}-\mathrm{BDNF})$; iv) $35 \mathrm{mmol} / \mathrm{l}$ glucose + $100 \mathrm{ng} / \mathrm{ml} \mathrm{BDNF}(\mathrm{HG}+\mathrm{M}-\mathrm{BDNF})$; and v) $35 \mathrm{mmol} / \mathrm{l}$ glucose $+125 \mathrm{ng} / \mathrm{ml} \mathrm{BDNF}$ (HG + H-BDNF). Following BDNF treatment (4 days), the cells were fixed in $4 \%(\mathrm{v} / \mathrm{v})$ neutral paraformaldehyde for $30 \mathrm{~min}$ and blocked with $3 \%$ peroxide-methanol at room temperature for endogenous peroxidase ablation. To block the non-specific binding sites, samples were incubated with $1 \%$ bovine serum albumin and $0.5 \%$ Triton $\mathrm{X}-100$ in phosphate-buffered saline (PBS) for $20 \mathrm{~min}$, followed by $10 \%$ normal goat serum in PBS for $20 \mathrm{~min}$. The expression of TrkB was then detected by immunostaining with a rabbit anti-mouse TrkB polyclonal antibody (1:50; Cell Signaling Technology, Inc., Danvers, MA, USA). Following sequential incubation with biotinylated secondary antibody and avidin-biotin-peroxidase reagents, the cells were stained with $0.5 \mathrm{mg} / \mathrm{ml}$ horseradish peroxidase (HRP) substrate solution $\left[0.05 \%\right.$ DAB and $0.03 \% \mathrm{H}_{2} \mathrm{O}_{2}$ prepared in Tris- $\mathrm{Cl}$ buffer ( $\mathrm{pH}$ 7.4)]. Next, the cells were counterstained with hematoxylin for $25 \mathrm{sec}$, followed by dehydration, clearing and mounting with neutral gums. The negative control group was subjected to the same protocol; however, the primary antibody was replaced by PBS. Histological assessments were performed as described previously (21).

MTT analysis. Retinal neuron cells were seeded in 96-well tissue culture plates $\left(1-3 \times 10^{4}\right.$ cells/well) and then treated with various combinations of glucose and BDNF as described. Next, 4 days following the addition of BDNF, thiazolyl blue tetrazolium bromide (MTT; $5 \mathrm{mg} / \mathrm{ml}$; Sigma-Aldrich) was added to each well and incubated for $4 \mathrm{~h}$. Formazan crystals formed from MTT by living cells were dissolved in DMSO and the formazan purple solution was detected using a spectrophotometer at $570 \mathrm{~nm}$. Inhibitory ratios were calculated using the following formula: [(control - sample)/control] x 100.

FACS analysis. FACS analysis was performed to detect the levels of apoptosis of retinal neurons using the Annexin V-FITC apoptosis detection kit (Bender MedSystems GmbH, Vienna, Austria) according to the manufacturer's instructions. Briefly, retinal neurons with various treatments were detached with $0.25 \%$ trypsin, followed by $10 \%$ fetal bovine serum to terminate the reaction. The cells were then collected and resuspended in $250 \mu \mathrm{l}$ binding buffer, followed by staining with Annexin V-FITC and propidium iodide solution for $15 \mathrm{~min}$ at room temperature in the dark. The samples were analyzed immediately using the FACSCalibur flow cytometer.

Western blot analysis. Retinal neurons were treated under the following conditions: i) $5.5 \mathrm{mmol} / \mathrm{l}$ glucose in basal medium (Ctr); ii) $5.5 \mathrm{mmol} / 1$ glucose $+100 \mathrm{ng} / \mathrm{ml}$ BDNF (Ctr + BDNF); iii) $35 \mathrm{mmol} / \mathrm{l}$ glucose $(\mathrm{HG})$; and iv) $35 \mathrm{mmol} / \mathrm{l}$ glucose $+100 \mathrm{ng} / \mathrm{ml} \mathrm{BDNF}(\mathrm{HG}+\mathrm{BDNF})$. The cells were then washed with D-PBS and lysed with cell lysis buffer [20 mmol/1 Tris-HCl ( $\mathrm{pH} 7.4), 150 \mathrm{mmol} / \mathrm{l} \mathrm{NaCl}, 1 \%$ Triton X-100, $0.1 \%$ SDS, $1 \%$ sodium deoxycholate, $1 \mathrm{mmol} / 1$ EDTA, $1 \mathrm{mmol} / 1$ phenylmethylsulfonyl fluoride, $1 \mu \mathrm{g} / \mathrm{ml}$ aprotinin and $\left.1 \mathrm{mmol} / 1 \mathrm{Na}_{3} \mathrm{VO}_{4}\right]$. Total proteins were exacted by centrifugation at $13,000 \mathrm{x} \mathrm{g}$ for $15 \mathrm{~min}$ and denatured in a $100^{\circ} \mathrm{C}$ water-bath for $5 \mathrm{~min}$. The concentration of total protein was measured using the Bradford method.

For western blot analysis, the proteins (25 $\mu \mathrm{g} /$ lane) were separated by electrophoresis using 10\% SDS-PAGE gels and then transferred onto polyvinylidene fluoride membranes. The membranes were blocked with blocking buffer (Tris-buffered saline, $5 \%$ nonfat dry milk and $0.1 \%$ Tween-20) prior to incubation with polyclonal antibodies against ERK, pERK, TrkB, pTrkB and monoclonal antibody against $\beta$-actin (all purchased from Cell Signaling Technology, Inc.). Following incubation with HRP-conjugated secondary antibody (Jackson ImmunoResearch Laboratories, Inc., West Grove, PA, USA) for $1 \mathrm{~h}$, ECL substrate (Pierce Biotechnology, Inc., Rockford, IL, USA) was used for detection. Images were captured and band density was analyzed using Bio-Rad Quantity One software (Bio-Rad, Hercules, CA, USA). Protein expression was normalized using $\beta$-actin as the control.

RNA isolation and RT-PCR. Total RNA was extracted using TRIzol reagent (Invitrogen Life Technologies, Carlsbad, CA, USA) according to the manufacturer's instructions. 

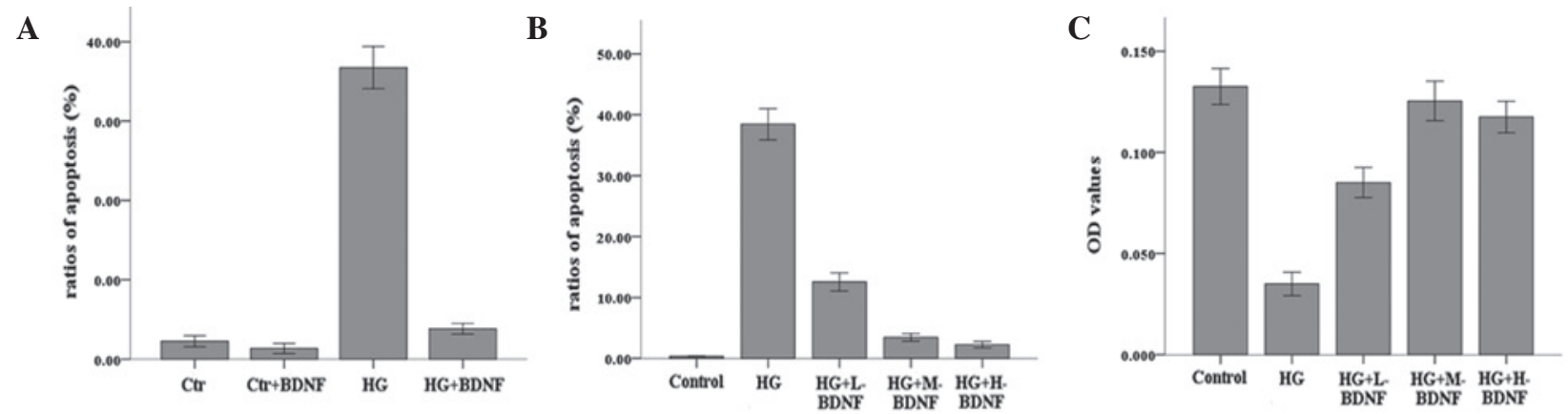

Figure 1. Protective effect of BDNF in retinal neurons under hyperglycemia. (A) FACS analysis demonstrated that hyperglycemia increases the rate of apoptosis, and BDNF protects cells and decreases the apoptosis ratio. (B) FACS analysis demonstrated that BDNF decreases the apoptosis ratio under hyperglycemia in a concentration-dependent manner. (C) MTT assay demonstrated that BDNF promotes cell survival under hyperglycemia in a concentration-dependent manner. Values are expressed as the mean $\pm \mathrm{SD}(\mathrm{n}=6)$. BDNF, brain-derived neurotrophic factor; OD, optical density; Ctr, $5.5 \mathrm{mmol} / \mathrm{l}$ glucose in basal medium; HG, $35 \mathrm{mmol} / 1$ glucose; HG + L-BDNF, 35 mmol/1 glucose + $75 \mathrm{ng} / \mathrm{ml}$ BDNF; HG + M-BDNF, 35 mmol/1 glucose + $100 \mathrm{ng} / \mathrm{ml}$ BDNF; HG + H-BDNF, 35 mmol/1 glucose $+125 \mathrm{ng} / \mathrm{ml}$ BDNF.

cDNA was synthesized from $1 \mu \mathrm{g}$ RNA via AMV Reverse Transcriptase (Promega Corporation, Madison, WI, USA). Semi-quantitative RT-PCR was performed to evaluate the mRNA levels of TrkB and ERK using the following primers: rat TrkB, sense 5'-TCTACAACGGAGCCATACTGAA-3' and antisense, 5'-CAGGCAGAATCCTACCACAGAG-3'; rat ERK, sense 5'-CCGCTCTATCCCAGACTTCACG-3' and antisense 5'-TGGTTTCCCACGGCTTCTACAC-3'; and rat $\beta$-actin, sense 5'-CCACTGCCGCATCCTCTT-3' and antisense 5'-CTCATCGTACTCCTGCTTGCT-3'.

RT-PCR products were analyzed on $1 \%$ agarose gels. Band density was analyzed using Band Leader 3.0 software. $\beta$-actin levels were used to normalize mRNA expression levels.

Statistical analysis. All values are presented as the mean \pm SEM. All data were analyzed using one-way ANOVA followed by post-hoc LSD multiple comparisons or the independent-samples t-test using SPSS version 13.0 (SPSS, Inc., Chicago, IL, USA). P $<0.05$ was considered to indicate a statistically significant difference.

\section{Results}

Protective effect of BDNF on retinal neurons under hyperglycemia. To assess the effects of hyperglycemia and the neurotrophic factor, BDNF, on the survival of neurons, levels of apoptosis were evaluated by FACS analysis. As demonstrated in Fig. 1A, the cells exposed to hyperglycemia exhibited higher levels of apoptosis, while BDNF was observed to markedly inhibit apoptosis mediated by hyperglycemia. In addition, the protective effects of BDNF on cells were demonstrated to be concentration dependent and the optimal concentration of BDNF was $100 \mathrm{ng} / \mathrm{ml}$. There was a positive correlation between the concentration of BDNF and the survival of neurons when the concentration was $<100 \mathrm{ng} / \mathrm{ml}$. MTT and FACS analysis results revealed that cell survival increased and levels of apoptosis decreased significantly as the concentration of BDNF increased (Fig. 1B and C). However, when cells were treated with $>100 \mathrm{ng} / \mathrm{ml}$ BDNF, cell survival and apoptosis were largely unaltered, indicating that the protective effect of BDNF had reached a plateau $(\mathrm{P}>0.05)$. These results indicate that hyperglycemia induces retinal neuron cell death and BDNF protects cells by decreasing levels of apoptosis in a concentration-dependent manner.

BDNF promotes the expression of TrkB in retinal neurons under hyperglycemia. To determine the mechanism by which BDNF protects retinal neurons from hyperglycemia, the endogenous levels of TrkB in cells were examined using immunohistochemistry. Compared with the control group, hyperglycemia treatment alone slightly elevated the expression of TrkB. However, TrkB expression increased markedly when cells were subjected to hyperglycemia combined with BDNF treatment (Fig. 2A and B). The effect of BDNF on TrkB expression was concentration dependent. Staining intensity was observed to increase with the concentration of BDNF (Fig. 2A and B). These results indicate that BDNF promotes the expression of TrkB in a concentration-dependent manner.

BDNF increases the mRNA levels of TrkB but not ERK in retinal neurons under hyperglycemia. To further confirm that BDNF promotes the expression of TrkB, RT-PCR was performed to examine mRNA levels. Following cell culture under normal or hyperglycemic conditions, in the presence or absence of BDNF (100 ng/ml), total RNA was extracted and TrkB mRNA levels were examined. Consistent with our previous results, hyperglycemia alone was observed to increase the expression of TrkB. The results also demonstrated that BDNF treatment alone did not alter TrkB levels; however, its expression increased markedly when cells were subjected to hyperglycemia in the presence of BDNF (Fig. 3A and B).

ERK is known to be located downstream of TrkB, therefore, the mRNA levels of ERK under various treatment conditions were analyzed. Neither hyperglycemia and/or BDNF treatment affected ERK mRNA levels (Fig. 3C and D). These results indicate that in retinal neurons under hyperglycemia, BDNF treatment elevated TrkB mRNA levels without affecting the mRNA levels of its downstream gene, ERK.

BDNF promotes phosphorylation of TrkB and ERK. Phosphorylation of TrkB is a key step for its activation. As 
A

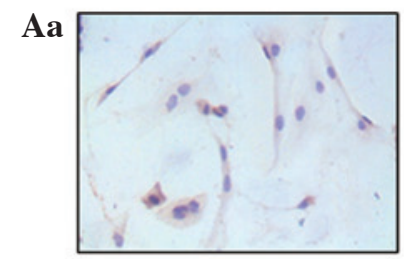

Ac

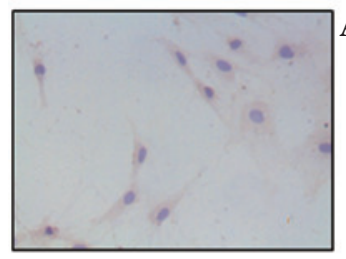

Ab

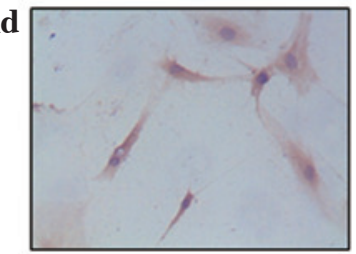

B

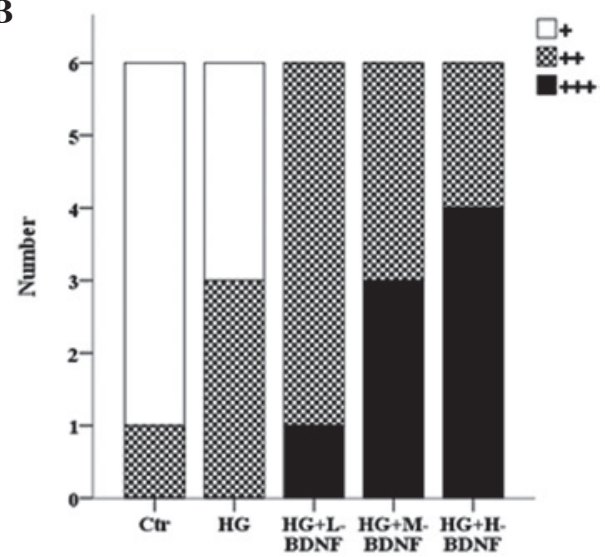

Figure 2. BDNF promotes the expression of TrkB in retinal neurons under hyperglycemia. (A) Immunohistochemistry. (Aa) Control; (Ab) HG; (Ac) HG + L-BDNF; (Ad) HG + M-BDNF; and (Ae) HG + H-BDNF. Images reveal that BDNF promotes TrkB expression. Representative images of 6 independent experiments are presented. (B) Quantification of Fig. 2A. BDNF, brain-derived neurotrophic factor; TrkB, tropomyosin-related kinase B; OD, optical density; Ctr, $5.5 \mathrm{mmol} / 1$ glucose in basal medium; HG, $35 \mathrm{mmol} / 1$ glucose; HG + L-BDNF, 35 mmol/1 glucose + $75 \mathrm{ng} / \mathrm{ml} \mathrm{BDNF}$; HG + M-BDNF, $35 \mathrm{mmol} / 1$ glucose + $100 \mathrm{ng} / \mathrm{ml}$ BDNF; HG + H-BDNF, 35 mmol/l glucose + $125 \mathrm{ng} / \mathrm{ml}$ BDNF.
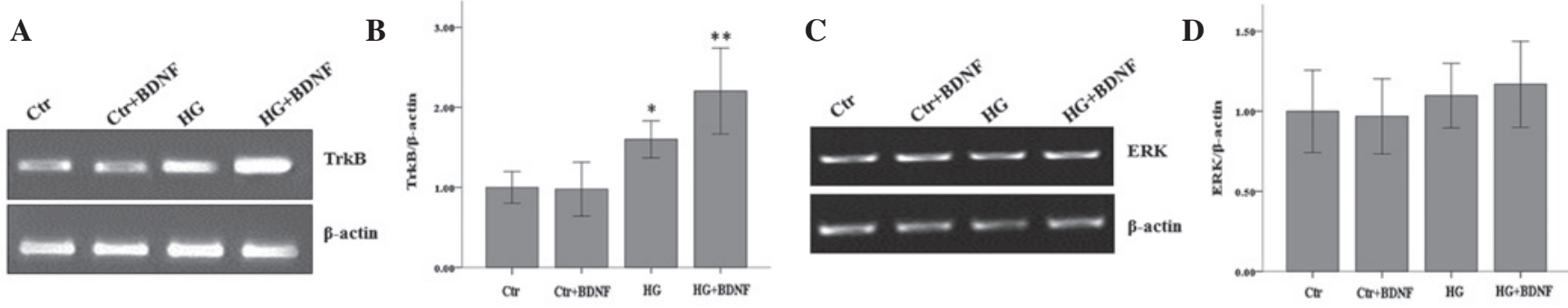

Figure 3. RT-PCR of TrkB and ERK mRNA expression. BDNF increases mRNA levels of TrkB but not ERK in retinal neurons under hyperglycemia. (A) mRNA levels of TrkB were elevated by BDNF in cells under hyperglycemia. (B) Quantification of Fig. 3A; ${ }^{*} \mathrm{P}<0.05$ and ${ }^{* *} \mathrm{P}<0.01$, vs. control. (C) mRNA levels of ERK were not affected by BDNF. (D) Quantification of Fig. 3C. BDNF, brain-derived neurotrophic factor; TrkB, tropomyosin-related kinase B; ERK, extracellular signal-regulated kinase; Ctr, $5.5 \mathrm{mmol} / 1$ glucose in basal medium; HG, $35 \mathrm{mmol} / 1$ glucose.
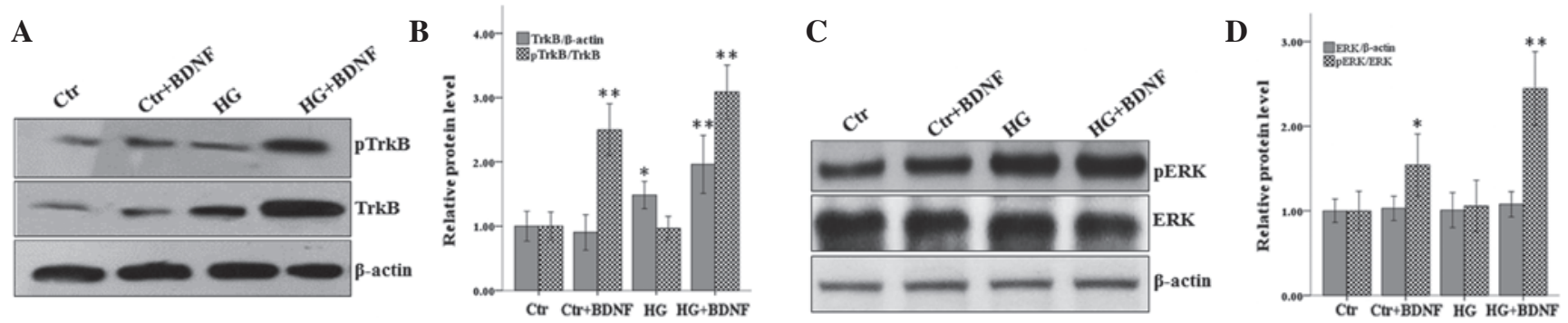

Figure 4. Western blot analysis of TrkB, pTrkB, ERK and pERK protein expression levels. BDNF promotes phosphorylation of TrkB and ERK. (A) BDNF increases the levels of total expression and phosphorylation of TrkB in cells under hyperglycemia. (B) Quantification of Fig. 4A; "P<0.05 and ${ }^{* *} \mathrm{P}<0.01$, vs. control. (C) BDNF increases phosphorylation but not total expression of ERK. (D) Quantification of Fig. 4B; " $\mathrm{P}<0.05$ and ${ }^{* *} \mathrm{P}<0.01$, vs. control. BDNF, brain-derived neurotrophic factor; TrkB, tropomyosin-related kinase B; Ctr, $5.5 \mathrm{mmol} / 1$ glucose in basal medium; HG, $35 \mathrm{mmol} / 1$ glucose.

BDNF was observed to increase the expression of $\operatorname{TrkB}$, the effect of BDNF on the phosphorylation of TrkB was determined. Cells were treated as described and western blot analysis was performed. As shown in Fig. 4, the results were consistent with the hypothesis that BDNF and hyperglycemia affected the expression levels of TrkB. In addition, BDNF increased the phosphorylation of TrkB markedly while hyperglycemia treatment had no effect. The rate of phosphorylation of TrkB also increased markedly when cells were subjected to hyperglycemia and treated with BDNF (Fig. 4A and B).
The phosphorylation of ERK was also analyzed in these cells. Consistent with the results of RT-PCR, BDNF and/or hyperglycemia did not affect the protein levels of ERK and the levels of phosphorylation of ERK were unchanged when the cells were subjected to hyperglycemia and treated with BDNF (Fig. 4C and D). However, treatment with BDNF alone was demonstrated to markedly increase the phosphorylation of ERK. These results indicate that BDNF but not hyperglycemia treatment promoted the increased phosphorylation of TrkB and ERK. 


\section{Discussion}

Previous studies have reported that BDNF and NT-4 are key factors for the survival of injured adult rat RGCs. The neuroprotective effect of BDNF and NT-4 was observed to be mediated by binding to the high affinity receptor, TrkB, expressed in RGCs (5). In addition, BDNF protects RGCs following acute high intraocular pressure by affecting the expression and phosphorylation of the TrkB receptor. However, to date, a limited number of studies have analyzed the protective effect of BDNF on retinal neurons exposed to hyperglycemia in vitro. In the present study, a model of primary hyperglycemia in retinal neurons was used to analyze this protective effect. BDNF was identified to increase the expression and phosphorylation of TrkB and protect neurons in the retina from hyperglycemia. In addition, phosphorylation of ERK was demonstrated to increase following BDNF treatment, indicating that ERK is a downstream effector of BDNF.

Consistent with previous studies, BDNF was observed to protect retinal neurons from hyperglycemia and this neuroprotective effect was concentration dependent. However, when the concentration was $>100 \mathrm{ng} / \mathrm{ml}$, the neuroprotective effect was not improved. An explanation for this observation may be that the number of TrkB receptors is the limiting factor and BDNF is saturated. Alternatively, the super-optimal concentration of BDNF may lead to inflammation and neuronal injury.

As the receptor of BDNF, TrkB is critical for its neuronprotective function. BDNF was shown to upregulate the expression of TrkB at the mRNA and protein levels in retinal neurons under conditions of hyperglycemia. Hyperglycemia also led to higher expression levels of TrkB, which was consistent with the findings of previous studies that mild brain injury differentially alters neurotrophin and neurotrophin receptor levels $(22,23)$. Although the levels of the TrkB receptor in neurons exposed to hyperglycemia was shown to increase significantly, apoptosis levels in these cells were still higher compared with the control. These results indicate that the higher expression of TrkB induced by hyperglycemia may be a compensatory mechanism for self-protection. As the phosphorylation of TrkB is critical for its activation, phosphorylation of TrkB was analyzed in cells under various treatment conditions. The results revealed that BDNF stimulated the phosphorylation of TrkB markedly while hyperglycemia had almost no effect (Fig. 4A and B).

Apoptosis is a form of programmed cell death that is important in multicellular organisms. Apoptosis is regulated by numerous proteins mainly associated with the canonical mitochondrial or endoplasmic reticulum signaling pathways. Previous studies have reported that normal RGCs constitutively express the antiapoptotic molecules, Akt, Cox-2 and $\mathrm{Mcl}-1$, while diabetes induced the expression of proapoptotic molecules, including mitochondrial proteins, cytochrome c and AIF (24). These observations indicate a marked correlation between apoptosis and neuronal survival; however, the mechanism of this process remains unclear (25-27). As apoptosis in diabetic neurons is associated with the lack of neurotrophic factors, in the present study, we hypothesized that adding extra neurotrophin may protect diabetic neurons. The results confirmed that BDNF protects retinal neurons from hyperglycemia by decreasing the rate of apoptosis. As a regulator of apoptosis, the Ras-MAPK/ERK pathway was previously reported to be downstream of TrkB. In the present study, ERK expression was detected in neurons exposed to hyperglycemia medium or treated with BDNF. The results revealed that hyperglycemia and/or BDNF did not affect the expression of ERK at the mRNA or protein levels. However, BDNF was demonstrated to markedly increase phosphorylation of ERK, while hyperglycemia treatment had no effect. These observations are consistent with the findings of a previous study (28). These results indicate that the ERK/MAPK signaling pathway is downstream of TrkB and BDNF. BDNF activates the TrkB-ERK/MAPK pathway and inhibits apoptosis, thus promoting the survival of neurons from hyperglycemia.

The results of the present study indicate that TrkB and ERK/MAPK are involved in neuroprotection against hyperglycemia induced by BDNF; however, further studies are required to determine the mechanism of this process. The results are likely to provide an important insight for further studies into the mechanism of BDNF protection of retinal neurons.

\section{Acknowledgements}

This study was supported by grants from the Health Department of Hunan Province (no. B2009-050) and the Science and Technology Program of Hunan Province (no. 2012FJ4077).

\section{References}

1. Barber AJ: A new view of diabetic retinopathy: a neurodegenerative disease of the eye. Prog Neuropsychopharmacol Biol Psychiatry 27: 283-290, 2003.

2. Lieth E, Barber AJ, Xu B, et al: Glial reactivity and impaired glutamate metabolism in short-term experimental diabetic retinopathy. Penn State Retina Research Group. Diabetes 47: 815-820, 1998.

3. Fernyhough P, Huang TJ and Verkhratsky A: Mechanism of mitochondrial dysfunction in diabetic sensory neuropathy. $\mathrm{J}$ Peripher Nerv Syst 8: 227-235, 2003.

4. Mey J and Thanos S: Intravitreal injections of neurotrophic factors support the survival of axotomized retinal ganglion cells in adult rats in vivo. Brain Res 602: 304-317, 1993.

5. Peinado-Ramón P, Salvador M, Villegas-Pérez MP and Vidal-Sanz M: Effects of axotomy and intraocular administration of NT-4, NT-3 and brain-derived neurotrophic factor on the survival of adult rat retinal ganglion cells. A quantitative in vivo study. Invest Ophthalmol Vis Sci 37: 489-500, 1996.

6. Pinzón-Duarte G, Arango-González B, Guenther E and Kohler K: Effects of brain-derived neurotrophic factor on cell survival, differentiation and patterning of neuronal connections and Müller glia cells in the developing retina. Eur J Neurosci 19: 1475-1484, 2004.

7. Seigel GM, Chiu L and Paxhia A: Inhibition of neuroretinal cell death by insulin-like growth factor- 1 and its analogs. Mol Vis 6: 157-163, 2000.

8. Müller A, Hauk TG and Fischer D: Astrocyte-derived CNTF switches mature RGCs to a regenerative state following inflammatory stimulation. Brain 130: 3308-3320, 2007.

9. Murphy JA, Franklin TB, Rafuse VF and Clarke DB: The neural cell adhesion molecule is necessary for normal adult retinal ganglion cell number and survival. Mol Cell Neurosci 36: 280-292, 2007.

10. Liu X, Clark AF and Wordinger RJ: Expression of ciliary neurotrophic factor (CNTF) and its tripartite receptor complex by cells of the human optic nerve head. Mol Vis 13: 758-763, 2007.

11. Yoshii A and Constantine-Paton M: Postsynaptic BDNF-TrkB signaling in synapse maturation, plasticity and disease. Dev Neurobiol 70: 304-322, 2010 
12. Hetman M, Kanning K, Cavanaugh JE and XiaZ: Neuroprotection by brain-derived neurotrophic factor is mediated by extracellular signal-regulated kinase and phosphatidylinositol 3-kinase. J Biol Chem 274: 22569-22580, 1999.

13. Almeida RD, Manadas BJ, Melo CV, et al: Neuroprotection by BDNF against glutamate-induced apoptotic cell death is mediated by ERK and PI3-kinase pathways. Cell Death Differ 12: 1329-1343, 2005.

14. Bonni A, Brunet A, West AE, Datta SR, Takasu MA and Greenberg ME: Cell survival promoted by the Ras-MAPK signaling pathway by transcription-dependent and -independent mechanisms. Science 286: 1358-1362, 1999.

15. Han BH and Holtzman DM: BDNF protects the neonatal brain from hypoxic-ischemic injury in vivo via the ERK pathway. J Neurosci 20: 5775-5781, 2000.

16. Satoh T, Nakatsuka D, Watanabe Y, Nagata I, Kikuchi H and Namura S: Neuroprotection by MAPK/ERK kinase inhibition with U0126 against oxidative stress in a mouse neuronal cell line and rat primary cultured cortical neurons. Neurosci Lett 288: $163-166,2000$

17. Jin K, Mao XO, Zhu Y and Greenberg DA: MEK and ERK protect hypoxic cortical neurons via phosphorylation of Bad. J Neurochem 80: 119-125, 2002.

18. Klöcker N, Kermer P, Weishaupt JH, Labes M, Ankerhold R and Bähr M: Brain-derived neurotrophic factor-mediated neuroprotection of adult rat retinal ganglion cells in vivo does not exclusively depend on phosphatidyl-inositol-3'-kinase/protein kinase B signaling. J Neurosci 20: 6962-6967, 2000.

19. Meng M, Zhiling W, Hui Z, Shengfu L, Dan Y and Jiping H: Cellular levels of TrkB and MAPK in the neuroprotective role of BDNF for embryonic rat cortical neurons against hypoxia in vitro. Int J Dev Neurosci 23: 515-521, 2005.
20. Song XY, Li F, Zhang FH, Zhong JH and Zhou XF: Peripherally-derived BDNF promotes regeneration of ascending sensory neurons after spinal cord injury. PLoS One 3: e1707, 2008.

21. Fromowitz FB, Viola MV, Chao S, et al: ras p21 expression in the progression of breast cancer. Hum Pathol 18: 1268-1275, 1987.

22. Hicks RR, Martin VB, Zhang L and Seroogy KB: Mild experimental brain injury differentially alters the expression of neurotrophin and neurotrophin receptor mRNAs in the hippocampus. Exp Neurol 160: 469-478, 1999.

23. Cui Q, Tang LS, Hu B, So KF and Yip HK: Expression of trkA, trkB and trkC in injured and regenerating retinal ganglion cells of adult rats. Invest Ophthalmol Vis Sci 43: 1954-1964, 2002

24. Abu El-Asrar AM, Dralands L, Missotten L and Geboes K: Expression of antiapoptotic and proapoptotic molecules in diabetic retinas. Eye (Lond) 21: 238-245, 2007.

25. Santiago AR, Cristóvão AJ, Santos PF, Carvalho CM and Ambrósio AF: High glucose induces caspase-independent cell death in retinal neural cells. Neurobiol Dis 25: 464-472, 2007.

26. Li YH, Zhuo YH, Lü L, et al: Caspase-dependent retinal ganglion cell apoptosis in the rat model of acute diabetes. Chin Med J (Engl) 121: 2566-2571, 2008.

27. Oshitari T, Yamamoto S, Hata N and Roy S: Mitochondria- and caspase-dependent cell death pathway involved in neuronal degeneration in diabetic retinopathy. Br J Ophthalmol 92: 552-556, 2008.

28. Omori K, Naruishi K, Nishimura F, Yamada-Naruishi H and Takashiba S: High glucose enhances interleukin-6-induced vascular endothelial growth factor 165 expression via activation of gp130-mediated p44/42 MAPK-CCAAT/enhancer binding protein signaling in gingival fibroblasts. J Biol Chem 279: 6643-6649, 2004. 